Research Paper

\title{
Integrative Oncology Outpatient Consultations: Long-Term Effects on Patient-Reported Symptoms and Quality of Life
}

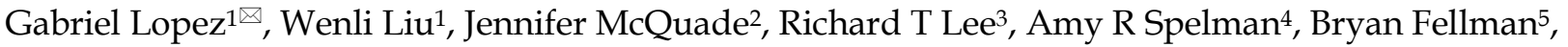 \\ Yisheng $\mathrm{Li}^{5}$, Eduardo Bruera ${ }^{1}$, Lorenzo Cohen ${ }^{1}$ \\ 1. Department of Palliative, Rehabilitation, and Integrative Medicine, The University of Texas MD Anderson Cancer Center; \\ 2. Department of Melanoma Medical Oncology, The University of Texas MD Anderson Cancer Center; \\ 3. Department of Medicine, University Hospitals and Case Western Reserve University; \\ 4. Department of Radiation Oncology, The University of Texas MD Anderson Cancer Center; \\ 5. Department of Biostatistics, The University of Texas MD Anderson Cancer Center. \\ $\bowtie$ Corresponding author: Gabriel Lopez \\ (C) Ivyspring International Publisher. This is an open access article distributed under the terms of the Creative Commons Attribution (CC BY-NC) license \\ (https://creativecommons.org/licenses/by-nc/4.0/). See http://ivyspring.com/terms for full terms and conditions.
}

Received: 2016.12.22; Accepted: 2017.03.21; Published: 2017.06 .03

\begin{abstract}
Background: Integrative oncology (IO) seeks to bring non-conventional approaches into conventional oncology care in an evidence-based, coordinated manner. Little is known about the effects of such consultations on patient-reported symptoms.

Methods: We reviewed data from patients referred for an 10 outpatient consultation between 2009 and 2013 , comparing the cohort of patients with at least one follow-up to the cohort with an initial consultation only. Assessments completed at initial and follow-up encounters included: complementary and alternative medicine (CAM) use questionnaire, Measure Yourself Concerns and Wellbeing (MYCaW), Edmonton Symptom Assessment Scale (ESAS; 10 symptoms, scale 0-10, 10 worst), and post-consultation satisfaction. ESAS individual items and global (GDS; score 0-90), physical (PHS, 0-60) and psychological (PSS, 0-20) distress scales were analyzed.

Results: 642 patients out of 2,474 (26\%) new patient 10 consultations had at least one follow-up encounter (mean 3.2; SD 1.8). Age, place of residence, and higher satisfaction were predictors of follow-up. Statistically significant improvement in symptoms between initial consult and follow-up were observed for depression, anxiety, well-being, and subscales of GDS and PSS (all p's > 0.01). For those with moderate to severe symptoms at their initial consult (ESAS scores $\geq 4$ ), we observed clinical response rates (improvement) of 49-75\% for all ESAS symptoms at follow-up.

Conclusions: Patients presenting for 10 follow-up had overall mild to moderate symptoms at baseline and stable symptom burden over time. Greatest improvements were observed for psychosocial symptoms, most pronounced for the subset of patients with moderate to severe symptoms at their initial consultation.
\end{abstract}

Key words: Integrative Oncology, Integrative Medicine, Patient Reported Outcomes, Edmonton Symptom Assessment System, Quality of Life, Complementary Approaches.

\section{Introduction}

An estimated $30-50 \%$ of cancer patients use complementary or alternative medicine (CAM).1,2,3, To assist with decision making in pursuing CAM approaches, patients commonly access a variety of resources that may or not offer evidence based recommendations. ${ }^{5}$ More recently, the term complementary and integrative medicine (CIM) has been used to describe an evidence-informed approach to pursuing these modalities.

Integrative medicine (IM) is a discipline that seeks to bring evidence-informed, non-conventional approaches into conventional medical care in a coordinated and safe manner. IM is increasingly becoming a part of health care services at academic centers across the United States and internationally, with over 60 member institutions forming the 
Academic Consortium for Integrative Medicine and Health $^{6}$ in the US, Canada and Mexico. Given the unique needs of cancer patients, most major National Cancer Institute (NCI) designated comprehensive cancer centers now have an IM program, although available services vary widely. ${ }^{7}$ The term integrative oncology (IO) is used to describe the application of integrative medicine to cancer care.

The University of Texas MD Anderson Cancer Center's Integrative Medicine Program was established in 1998. The clinical center offers group programs as well as individual services including oncology massage, acupuncture, physical therapy, nutrition, meditation, health psychology, and music therapy. Our clinical model is based on the bio-psychosocial model of care, thoughtfully incorporating CIM approaches that address physical, psycho-spiritual, and social dimensions of health. ${ }^{8}$

The integrative oncology physician consultation serves as the foundation for developing an integrative care plan that includes complementary health approaches and lifestyle changes. Patients are seen in follow-up to review the integrative care plan, making modifications as needed based on their unique cancer trajectory.

Although the literature provides a wealth of information on patient interest in and use of CIM approaches, ${ }^{1,2}$ there is a limited understanding of the impact of physician guided integrative care plans on self-reported symptoms and characteristics of patients who follow-up with integrative oncology. $., 9,10$ To address this lack of knowledge, we have previously examined the demographics, disease characteristics, reason for consultation, symptom burden, and satisfaction of an initial IO consultation at a major US cancer center. ${ }^{11}$ The current manuscript examines the reasons for follow-up, the characteristics of those patients, and changes in self-reported symptoms and quality of life.

\section{Methods}

This study was conducted at the University of Texas MD Anderson Cancer Center's Integrative Medicine Center between September 2009 and December 2013. All patients presenting for an IO encounter (initial consultation and/or follow-up encounter) were asked to complete a series of questionnaires as part of an IRB approved protocol; only patients with age $>18$ were included in this analysis. Immediately prior to their initial consultation, patients completed a complementary and alternative medicine (CAM) use questionnaire, the Measure Yourself Concerns and Wellbeing (MYCaW) and Edmonton Symptom Assessment Scale (ESAS). At follow-up, patients completed the MYCaW and ESAS. Immediately after the initial consultation and follow-up encounter, patients were asked how well their top two MYCaW concerns were addressed and a single question regarding overall satisfaction with the encounter.

\section{Intervention}

All IO initial consultation and follow-up encounters included a comprehensive assessment, responding to both patient self-reported data made available to the clinician immediately prior to the encounter and patient concerns elicited through narrative during the encounter. As part of the initial consultation, the majority of patients met with an advanced practice provider immediately prior to meeting with the physician. During the initial encounter with the physician and Advanced Practice Provider, each patient is evaluated comprehensively and referrals are made to our other services according to the individual's physical, mind-body, or social needs. An integrative care plan may include acupuncture or massage for symptom control; health psychology, meditation, or music therapy for psychological distress; counseling on healthy lifestyle behaviors and referral to nutrition and physical therapy; or discussion of risk and evidence-base for herb/supplements or alternative treatments being pursued or considered by patients. Patients are recommended follow-up based on goals of their integrative care plan. For the follow-up encounter, patients met with a physician, advanced practice provider, or both as an opportunity to review prior recommendations and discuss any new concerns.

\section{Measures}

All instruments were completed using paper forms and then entered into an electronic database for analysis. Patient demographics and clinical data were extracted from the medical record. Our internally developed CAM use questionnaire asked patients about CAM use in the year preceding their consultation; CAM items were based on the NIH-National Center for Complementary and Integrative Health $(\mathrm{NCCIH})$ complementary health categories of natural products, mind and body approaches, and other complementary health approaches. This questionnaire was administered for initial consultations only from Jan 1, 2013 through Dec 31, 2013.

\section{Measure Yourself Concerns and Wellbeing (MYCaW)}

At each encounter (initial consultation and follow-up), patients completed a modified version of the MYCaW questionnaire. ${ }^{12}$ Patients identified the top two concerns for their integrative medicine encounter from a list of available topic areas including 
integrative/holistic approach, herb/supplements, diet/nutrition, pain, overall health and stress/anxiety, as well as an "other" category. In addition, patients were asked to rate the importance of each concern on a scale from $0-10$, with 10 being highly important.

\section{Edmonton Symptom Assessment Scale (ESAS)}

Patient symptom burden was assessed using the ESAS. ${ }^{13}$ Patients were asked to report on ten symptoms: pain, fatigue, nausea, depression, anxiety, drowsiness, loss of appetite, decreased sense of well-being, shortness of breath, and sleep-as experienced in the prior 24 hours on a numeric scale of 0 to 10, 10 being the worst. Subscale scoring was as follows: global distress score (GDS, 0-90) sum of pain, fatigue, nausea, drowsiness, appetite, shortness of breath, anxiety, depression, and well-being scores; physical distress score (PHS, 0-60) sum of pain, fatigue, nausea, drowsiness, appetite, and shortness of breath; psychological distress score (PSS, 0-20) sum of anxiety and depression. A reduction of 1 is viewed as a clinically significant improvement in that symptom. ${ }^{14}$

\section{Satisfaction}

Overall satisfaction with the each encounter (initial and follow-up) was assessed on a 0 to 10 scale [0-1 very dissatisfied, 2-4 dissatisfied, 5 neutral , 6-8 satisfied, 9-10 very satisfied].

\section{Statistical Analyses}

Summary statistics were used to describe demographics, QOL questionnaires and reason for consultation. We assessed whether there were differences in demographic characteristics of subjects who only came for an initial consultation versus those who came for at least one follow-up using a t-test for age and chi-squared test for categorical variables. Baseline (at initial consultation) and follow-up ESAS scores were compared using a Wilcoxon signed-rank test. Clinically significant reduction for individual ESAS symptoms were a priori defined as a symptom score reduction of $\geq 1,14$ and reduction of GDS $\geq 3$, PHS $\geq 2$, and PSS $\geq 2$ for the ESAS subscales ${ }^{15}$ as has been previously reported. Response rate was calculated for patients with baseline symptom score $\geq$ 4 and subset analyses conducted as above. To compare the MYCaW reasons for initial consultation versus follow-up, a chi-squared test was conducted. Frequencies of types of clinical services after the initial consultation were calculated to determine the most frequent services used after the initial consultation. Since this is an exploratory study to generate hypothesis for future studies, all testing was performed at a 0.05 two-sided significance level, without adjusting for multiple testing.

\section{Results}

\section{Patient characteristics}

Between September 1, 2009 and December 31, 2013, 2474 patients had an initial consultation and 642 $(26 \%)$ had at least one follow-up encounter following their initial consultation [Table 1]. Mean time to first follow up was 99.1 days (SD 146.6, range 1-1446). We compared demographic and medical characteristics of patients with only an initial consultation to those with at least one follow-up. Patients who followed-up were slightly older (57.2 vs. 55.5) and more likely to live locally. No significant differences were observed for gender, race, disease type, stage or prior CAM use.

Table 1. Comparison of demographic characteristics between initial consultation cohort versus follow-up cohort

\begin{tabular}{|c|c|c|c|c|c|}
\hline \multirow[t]{2}{*}{ Characteristic } & \multicolumn{2}{|c|}{$\begin{array}{l}\text { Initial consult }(\mathrm{N}= \\
1832)\end{array}$} & \multicolumn{2}{|c|}{$\begin{array}{l}\text { Follow-Up }(\mathrm{N}= \\
642)\end{array}$} & \multirow[t]{2}{*}{$\begin{array}{l}\text { p-valu } \\
\text { e }\end{array}$} \\
\hline & $\mathrm{N}$ & $\%$ & $\mathrm{~N}$ & $\%$ & \\
\hline \multicolumn{5}{|l|}{ Age (yrs) } & 0.005 \\
\hline $\mathrm{N}$ & \multicolumn{2}{|l|}{1754} & \multicolumn{2}{|l|}{623} & \\
\hline Mean (SD) & \multicolumn{2}{|l|}{55.5 (12.7) } & \multicolumn{2}{|c|}{$57.2(12.2)$} & \\
\hline Median (Min-Max) & \multicolumn{2}{|l|}{$(18.1-90.1)$} & \multicolumn{2}{|c|}{$(18.8-90.6)$} & \\
\hline \multicolumn{5}{|l|}{ Gender } & 0.160 \\
\hline Female & 1194 & 68.1 & 443 & 71.1 & \\
\hline Male & 560 & 31.9 & 180 & 28.9 & \\
\hline \multicolumn{5}{|l|}{ Race } & 0.583 \\
\hline White & 1324 & 75.5 & 456 & 73.2 & \\
\hline Spanish Surname & 173 & 9.9 & 67 & 10.8 & \\
\hline Black & 119 & 6.8 & 51 & 8.2 & \\
\hline Other & 137 & 7.8 & 49 & 7.9 & \\
\hline \multicolumn{5}{|l|}{ Residence } & 0.001 \\
\hline Harris County & 483 & 27.5 & 223 & 35.8 & \\
\hline 7 Surrounding Counties & 204 & 11.6 & 80 & 12.8 & \\
\hline Rest of Texas & 362 & 20.6 & 121 & 19.4 & \\
\hline Rest of US & 647 & 36.9 & 178 & 28.6 & \\
\hline International & 58 & 3.3 & 21 & 3.4 & \\
\hline \multicolumn{5}{|l|}{ Disease } & 0.127 \\
\hline Breast & 486 & 27.7 & 210 & 33.7 & \\
\hline Gastrointestinal & 230 & 13.1 & 82 & 13.2 & \\
\hline Genitourinary & 87 & 5.0 & 29 & 4.7 & \\
\hline Gynecologic & 112 & 6.4 & 32 & 5.1 & \\
\hline Leukemia & 31 & 1.8 & 10 & 1.6 & \\
\hline Lymphoma/Myeloma & 92 & 5.2 & 33 & 5.3 & \\
\hline Sarcoma & 83 & 4.7 & 19 & 3.0 & \\
\hline $\begin{array}{l}\text { Skin (including } \\
\text { melanoma) }\end{array}$ & 55 & 3.1 & 16 & 2.6 & \\
\hline $\begin{array}{l}\text { Thoracic/Head and } \\
\text { Neck }\end{array}$ & 159 & 9.1 & 40 & 6.4 & \\
\hline Multiple & 337 & 19.2 & 127 & 20.4 & \\
\hline Other & 82 & 4.7 & 25 & 4.0 & \\
\hline Stage & & & & & 0.501 \\
\hline Local & 872 & 61.8 & 298 & 63.5 & \\
\hline Advanced & 539 & 38.2 & 171 & 36.5 & \\
\hline \multirow[t]{2}{*}{ Prior CAM use } & & & & & 0.851 \\
\hline & 518 & 29.5 & 193 & 30.9 & \\
\hline
\end{tabular}

\section{Patient interests (MyCAW) \& Satisfaction}

Patients completed the MyCAW at initial consultation and follow-up [Table S1]. Comparing the initial consultation only and follow-up cohorts, patients in both groups had an equally high interest in 
topics of integrative/holistic approach and diet/nutrition. Those that presented for an initial consultation only had a significantly higher interest in discussing herbs \& supplements $(35.2 \%$ vs $29.9 \%$, $\mathrm{p}=0.020)$ and hot flashes ( $4 \%$ vs $2.1 \%, \mathrm{p}=0.032)$, whereas patients whose concern was neuropathy were more likely to follow-up $(12.4 \%$ vs $7.9 \%$, $\mathrm{p}=0.001$ ). Again, comparing initial consultation only and follow-up cohorts, patient interest in discussing their top two concerns was high $(>8$, out of possible 10; from post-encounter satisfaction item) and there were no differences observed in the importance of how well concern 1 or 2 were addressed. Patients in the follow-up cohort were significantly more satisfied with their initial consultation (9.6 versus 9.4, $\mathrm{p}=0.012$ ), yet the absolute value difference was small.

\section{ESAS scores}

\section{Initial consultation cohort versus follow-up cohort}

Compared to the cohort with an initial consultation only (initial consultation cohort), the follow-up cohort reported statistically higher anxiety (3.4 vs 3.1, p=0.015) and PSS (5.6 vs 5.2, $\mathrm{p}=0.049$ ) at initial visit; however, these differences did not reach clinical significance [Table 2].

Table 2. Comparison of Baseline ESAS between initial consultation cohort versus follow-up cohort

\begin{tabular}{llllll}
\hline \multirow{2}{*}{ ESAS } & \multicolumn{4}{l}{ Initial Consultation cohort Follow-Up cohort } & p-value \\
\cline { 2 - 5 } & $\mathbf{N}$ & Mean (SD) & N & Mean (SD) & \\
\hline Pain & 1744 & $2.6(2.8)$ & 614 & $2.6(2.7)$ & 0.614 \\
Fatigue & 1744 & $4.0(2.8)$ & 617 & $4.0(2.8)$ & 0.725 \\
Nauseated & 1731 & $1.0(2.0)$ & 613 & $1.0(1.9)$ & 0.304 \\
Sleep & 1716 & $4.2(2.9)$ & 600 & $4.1(2.8)$ & 0.721 \\
Shortness of breath & 1731 & $1.4(2.2)$ & 601 & $1.4(2.2)$ & 0.745 \\
Appetite & 1738 & $2.8(2.8)$ & 615 & $2.7(2.8)$ & 0.948 \\
Drowsy & 1726 & $2.3(2.7)$ & 613 & $2.5(2.7)$ & 0.086 \\
Depressed & 1741 & $2.2(2.6)$ & 611 & $2.2(2.6)$ & 0.456 \\
Anxious & 1751 & $3.1(2.9)$ & 616 & $3.4(2.9)$ & 0.015 \\
Well-being & 1732 & $3.8(2.6)$ & 607 & $3.8(2.6)$ & 0.950 \\
PHS* & 1636 & $13.9(10.4)$ & 573 & $14.1(10.7)$ & 0.837 \\
PSS & 1731 & $5.2(5.1)$ & 609 & $5.6(5.1)$ & 0.049 \\
GDS $^{*}$ & 1600 & $22.9(15.5)$ & 558 & $23.3(15.7)$ & 0.571 \\
\hline
\end{tabular}

*GDS (Global distress score) equals sum of pain, fatigue, nausea, depression, anxiety, drowsiness, appetite, well-being, and shortness of breath (total score 0-90); PHS (physical distress score) equals sum of pain, fatigue, nausea, drowsiness, appetite, and shortness of breath (total 0-60); and PSS (psychological distress score) equals sum of depression and anxiety.

\section{Follow-up cohort}

Examining the follow-up cohort $(\mathrm{n}=642)$, highest symptom scores at first follow-up included sleep (3.9), fatigue (3.8), and well-being (3.5) [Table 3]. Statistically significant improvement in symptoms between initial and follow-up were observed for depression $(-0.2, \mathrm{p}=0.009)$, anxiety $(-0.7, \mathrm{p}=0.001)$, well-being $(-0.3, p=0.004)$ and subscales of PSS (-0.9, $\mathrm{p}=0.001)$ and GDS (-1.6, $\mathrm{p}=0.010)$. No individual ESAS symptom or subscale change achieved clinical significance [Table 3]. ${ }^{14}$ The most frequent clinical services accessed at our integrative medicine center included acupuncture and massage with a greater percentage of the patients who attended a follow-up appointment using these and other services [Table 4]. Overall, patients who had at least one physician follow-up visit (follow-up cohort) were more likely to access additional clinical services.

Table 3. Follow-up cohort: ESAS scores at initial consultation and first follow-up*

\begin{tabular}{|c|c|c|c|c|c|}
\hline \multirow[t]{2}{*}{ ESAS } & \multicolumn{2}{|c|}{ Initial consultation } & \multicolumn{2}{|c|}{ Follow-Up } & \multirow[t]{2}{*}{ p-value } \\
\hline & $\mathbf{N}$ & Mean (SD) & $\mathbf{N}$ & Mean (SD) & \\
\hline Pain & 558 & $2.6(2.7)$ & 558 & $2.7(2.8)$ & 0.728 \\
\hline Fatigue & 559 & $4.0(2.8)$ & 559 & $3.8(2.9)$ & 0.189 \\
\hline Nauseated & 555 & $0.9(1.8)$ & 555 & $0.9(1.7)$ & 0.342 \\
\hline Sleep & 532 & $4.1(2.8)$ & 532 & $3.9(2.8)$ & 0.074 \\
\hline Shortness of breath & 535 & $1.4(2.2)$ & 535 & $1.3(2.1)$ & 0.586 \\
\hline Appetite & 548 & $2.8(2.8)$ & 548 & $2.6(2.8)$ & 0.082 \\
\hline Drowsy & 550 & $2.5(2.7)$ & 550 & $2.4(2.6)$ & 0.459 \\
\hline Depressed & 547 & $2.2(2.7)$ & 547 & $2.0(2.6)$ & 0.009 \\
\hline Anxious & 556 & $3.4(2.9)$ & 556 & $2.7(2.7)$ & 0.001 \\
\hline Well-being & 542 & $3.8(2.6)$ & 542 & $3.5(2.5)$ & 0.004 \\
\hline PHS+ & 490 & $14.0(10.6)$ & 490 & $13.5(10.3)$ & 0.283 \\
\hline PSSt & 543 & $5.6(5.2)$ & 543 & $4.7(4.9)$ & 0.001 \\
\hline GDSt & 459 & $23.4(15.8)$ & 459 & $21.8(15.2)$ & 0.010 \\
\hline \multicolumn{6}{|c|}{$\begin{array}{l}\text { * only evaluated subjects with complete data at both initial consultation and first } \\
\text { follow-up }\end{array}$} \\
\hline \multicolumn{6}{|c|}{$\begin{array}{l}\text { + GDS (Global distress score) equals sum of pain, fatigue, nausea, depression, } \\
\text { anxiety, drowsiness, appetite, well-being, and shortness of breath (total score 0-90); } \\
\text { PHS (physical distress score) equals sum of pain, fatigue, nausea, drowsiness, } \\
\text { appetite, and shortness of breath (total 0-60); and PSS (psychological distress score) } \\
\text { equals sum of depression and anxiety. }\end{array}$} \\
\hline
\end{tabular}

Table 4. Clinic service visits after initial consultation

\begin{tabular}{llllll}
\hline Clinic Service & \multicolumn{2}{l}{$\begin{array}{l}\text { Initial only cohort } \\
(\mathbf{N}=\mathbf{1 8 3 2})\end{array}$} & $\begin{array}{l}\text { Follow-up cohort } \\
(\mathbf{N}=\mathbf{6 4 2})\end{array}$ & p-value \\
\cline { 2 - 5 } & $\mathbf{N}^{*}$ & $\mathbf{0}$ & $\mathbf{N}^{*}$ & $\mathbf{\%}$ & \\
\hline Acupuncture & 109 & 5.95 & 55 & 8.57 & 0.022 \\
Dietician & 40 & 2.18 & 10 & 1.56 & 0.332 \\
Massage & 71 & 3.88 & 43 & 6.7 & 0.003 \\
Meditation & 22 & 1.2 & 19 & 2.86 & 0.003 \\
Music & 12 & 0.66 & 14 & 2.18 & 0.001 \\
Physical Therapy & 31 & 1.69 & 14 & 2.18 & 0.425 \\
\hline
\end{tabular}

${ }^{*} \mathrm{~N}$ represents unique patients accessing a service; each patient may have had more than one visit for each clinical service

\section{Subgroup of follow-up cohort with moderate to high symptom burden at initial presentation: ESAS scores $\geq 4$}

Long-term effects on patient-reported outcomes may be observed when comparing symptom burden at consultation with re-assessment at follow up. ${ }^{15} \mathrm{We}$ selected for patients with moderate to high symptom burden at initial presentation (ESAS symptom scores $\geq 4$ ) to increase our ability to detect clinically significant changes over time.14,16 In order to better link the changes in symptom reports to the advice that 
was provided during the initial consultation, we limited our examination to patients who followed-up within 30 days of their initial consultation. For the subset of patients with symptom scores $\geq 4$ at baseline and follow-up within 30 days of the initial consultation, we observed statistically and clinically significant reductions in all individual ESAS symptoms, with response rates ( $\geq 1$ point reduction) ranging from $49.0 \%$ to $75.0 \%$ [Table 5]. Of the ESAS subscales, only PSS revealed a clinically significant change (2.17) with a response rate of $56.3 \%$.

Table 5. Response rate for follow-up cohort when baseline ESAS symptom score was $\geq 4$ (restricted to visits within 30 days of each other)

\begin{tabular}{|c|c|c|}
\hline ESAS & $\begin{array}{l}\mathrm{N}^{*} \text { Clinical Response Rates ** } \\
\%(95 \% \mathrm{CI})\end{array}$ & Mean Changet (SD) \\
\hline Pain & $4949.0(34.4-63.7)$ & $1.10(2.49)$ \\
\hline Fatigue & $7254.2(42.0-66.0)$ & $1.19(2.42)$ \\
\hline Nauseated & $2075.0(50.9-91.3)$ & $2.75(3.29)$ \\
\hline Sleep & $6553.9(41.0-66.3)$ & $1.55(2.88)$ \\
\hline Shortness of breath & $2669.2(48.2-85.7)$ & $2.19(2.56)$ \\
\hline Appetite & $5552.7(39.0-66.4)$ & $1.07(3.07)$ \\
\hline Drowsy & $4656.5(41.1$ - 71.1) & $1.54(3.05)$ \\
\hline Depressed & $3961.5(44.6$ - 76.6$)$ & $1.74(3.06)$ \\
\hline Anxious & $5470.4(56.4-82.0)$ & $1.76(2.49)$ \\
\hline Well-being & $6662.1(49.3-73.8)$ & $1.45(2.86)$ \\
\hline PHSH & $9050.0(39.3-60.7)$ & $0.67(9.96)$ \\
\hline PSSH & $8056.3(44.7-67.3)$ & $2.17(4.43)$ \\
\hline GDSH & $9452.1(41.6-62.5)$ & $2.04(12.53)$ \\
\hline
\end{tabular}

* $\mathrm{N}$ represents the number of baseline scores greater than or equal to 4 with a follow-up encounter within the 30 day time frame.

** Defined as a 1-point decrease or more in score on the ESAS individual symptoms of pain through well-being; 2-point decrease or more in score on the ESAS PSS; 3-point decrease or more in score on the ESAS PHS and GDS

+Difference between score at baseline and follow-up (positive difference implies lower score at follow-up, i.e. improvement)

+ GDS (Global distress score) equals sum of pain, fatigue, nausea, depression, anxiety, drowsiness, appetite, well-being, and shortness of breath (total score 0-90); PHS (physical distress score) equals sum of pain, fatigue, nausea, drowsiness, appetite, and shortness of breath (total 0-60); and PSS (psychological distress score) equals sum of depression and anxiety.

\section{Discussion}

Patients present to an integrative oncology consultation with a diversity of interests/concerns, which they rate at a high level of importance. Through a thoughtful evaluation of patient symptoms and interests, the initial consultation aims at creating an integrative care plan that may or may not include referral to additional integrative clinical services. Although a majority of patients do not return for follow-up, those who do continue to rate their concerns on follow-up at a high level of importance. Those who did return were older, more likely to live in close proximity to the cancer center, and reported higher anxiety and psychological distress at initial consultation than those who did not attend a follow-up appointment.

Our findings are in contrast to palliative care populations who have an overall higher symptom burden at baseline ${ }^{16}$ and those who return for follow-up are those with the highest baseline psychosocial symptom burden. ${ }^{15}$ Our findings suggest that the population returning for an integrative oncology follow-up has a unique symptom and interest profile. The areas of highest interest on follow-up included an integrative approach, herbs \& supplements, and diet/nutrition. This suggests patients are seeking continued counseling and support for their integrative care plan rather than focusing on the management of a specific symptom. Those most satisfied with their baseline encounter were more likely to return for follow-up.

For the follow-up cohort, although we observed improvements in symptoms of depression, anxiety, well-being and subscales of PSS and GDS, mean follow-up scores for individuals symptoms of $<4$ suggested overall low symptom burden at follow-up [Table 3]. On review of symptoms at initial consultation for the follow-up cohort, only sleep (4.1) achieved a moderate $(\geq 4)$ level of intensity. Of note, only the ESAS subscale of PSS revealed clinically significant change, driven by statistically significant change observed in individual symptoms of anxiety and depression. Overall, there were statistically significant reductions in a number of individual symptoms, but the changes did not reach clinical significance, suggesting a stable symptom burden from consultation to follow-up for our patient population. Examination of the subgroup with moderate to severe symptom burden at baseline did reveal statistically and clinically significant changes in symptom reports over time, with highest response rate and greatest mean symptom improvement in those reporting nausea [Table 5]. Of the ESAS subscales, only change in mean PSS demonstrated a clinically significant change in the higher symptom burden subgroup. Even so, greater than half the patients reported a clinical improvement on all symptoms and subscales, with pain being the lowest and still benefiting $49 \%$ of patients. Our findings suggest that the IO consultation may be beneficial at reducing psychosocial symptoms, and especially useful for patients reporting moderate symptom burden at presentation, as the majority reported clinically significant improvements on multiple outcomes within 1 month.

There were some differences between the patients who attended the follow-up consultation and those who did not. Those who came for initial consultation only reported lower levels of anxiety and psychosocial distress than those in the follow-up cohort. On examination of baseline patient concerns in the initial consultation only and follow-up cohorts, 
integrative approach and herbs \& supplements were the top two areas of interest, not psychosocial symptoms. Interestingly, although patients are not recognizing psychosocial symptoms of anxiety and depression as their top concerns, these are the symptoms most impacted by an integrative oncology consultation.

There are a number of limitations to this clinic-based study. Our study was retrospective in nature and there is no comparison with which to compare the patients at follow-up. We therefore cannot solely attribute changes observed in symptom reporting to the consultation versus simply the passage of time or regression to the mean. Although we determined that a 30 day follow-up period would better capture the effects of our interventions on patient self-reported symptoms and quality of life, we could not control for patients seeking care outside of our center from either conventional or complementary health providers during this time period. Such interventions could have meaningful effects on symptom reported by patients. Our approach has been used in previous retrospective analyses looking at the long term effects of clinical interventions on symptom reporting. ${ }^{15,} 18$ Additional limitations include that the patient population was also a convenience sample of patients coming for an IO consultation within a major medical center and may not be representative of the larger population of patients seeking and using IO.

\section{Conclusion}

Overall, an initial integrative oncology consultation resulted in clinically meaningful improvements in psychosocial symptoms for patients who returned for follow-up. Greater overall impact across all symptoms was observed for those reporting a moderate to high symptom burden at initial presentation. Patients were very satisfied with the encounter at initial consultation and at follow-up, with their top concerns being addressed to their satisfaction. Future prospective studies are needed to determine which components of the integrative oncology consultation and subsequent IM clinical services are most likely to achieve clinically significant effects on physical and psychosocial aspects of quality of life.

\section{Supplementary Material}

Table S1. http://www.jcancer.org/v08p1640s1.pdf

\section{Acknowledgement}

The research was funded in part by support from the Duncan Family Institute. This research is also supported in part by the National Institutes of Health
(NIH) through M. D. Anderson's Cancer Center Support Grant CA016672.

\section{Author Contributions}

Gabriel Lopez: Conceptualization, investigation, writing - original draft; writing - review and editing, supervision, resources, investigation, data curation, visualization, project administration. Wenli Liu: writing - review and editing. Jennifer McQuade: writing - review and editing. Richard T Lee: writing review and editing. Amy R Spelman: Data curation, writing - review and editing. Bryan Fellman and Yisheng Li: methodology, data curation, formal analysis, writing - review and editing. Eduardo Bruera: Conceptualization, writing, review, editing. Lorenzo Cohen: Conceptualization, writing - review and editing, resources, supervision.

All authors have read and approved the manuscript. This manuscript is not under consideration elsewhere. There are no conflicts of interest and/or financial disclosures from any authors. The study was conducted as part of an IRB approved protocol.

\section{Competing Interests}

The authors have declared that no competing interest exists.

\section{References}

1. Gansler T, Kaw C, Crammer C, et al. A population-based study of prevalence of complementary methods use by cancer survivors: A report from the American Cancer Society's studies of cancer survivors. Cancer. 2008; 113:1048-1057.

2. Horneber M, Bueschel G, Dennert G, et al. How many cancer patients use complementary and alternative medicine: a systematic review and metaanalysis. Integr Cancer Ther. 2012; 11(3):187-203.

3. Girgis A, Adams J, Sibbritt D. The use of complementary and alternative therapies by patients with cancer. Oncol Res. 2005; 15: 281-289.

4. Richardson MA, Sanders T, Palmer JL, et al. Complementary/alternative medicine use in a comprehensive cancer center and the implications for oncology. Journal of Clinical Oncology, vol. 18, no. 13, pp. 2505-2514, 2000.

5. Deng G, and Cassileth B. Complementary or alternative medicine in cancer care-myths and realities. Nat Rev Clin Oncol. 2013 Nov;10(11):656-64.

6. [Internet] http://www.imconsortium.org/members/members.cfm

7. Seely DM, Weeks LC, and Young S. A systematic review of integrative oncology programs. Current Oncology. 2012; 19(6): e436-61.

8. Engle GL. The need for a new medical model: a challenge for biomedicine. Science 1977; 196:129-136.

9. Frenkel M, Cohen L, Peterson N, et al. Integrative medicine consultation service in a comprehensive cancer center: findings and outcomes. Integr Cancer Ther. 2010 Sep; 9(3):276-83.

10. Ben-Arye E, Schiff E, Zollman C, et al. Integrating complementary medicine in supportive cancer care models across four continents. Med Oncol. 2013 Jun;30(2): 511.

11. Lopez G, McQuade J, Cohen L, et al. Integrative Oncology Physician Consultations at a Comprehensive Cancer Center: Analysis of Demographic, Clinical and Patient Reported Outcomes. J Cancer 2017; 8(3):395-402.

12. Paterson $C$, Thomas $K$, Manassec A, et al. Measure Yourself Concerns and Wellbeing (MYCaW): An individualised questionnaire for evaluating outcome in cancer support care that includes complementary therapies. Complementary Therapies in Medicine. 2007; 15:38-45.

13. Bruera E, Kuehn N, Miller MJ, et al. The Edmonton Symptom Assessment System (ESAS): a simple method for the assessment of palliative care patients. J Palliat Care. 1991;7(2):6-9.

14. Hui $D$, Shamieh $O$, Eduardo Palva $C$, et al. Minimal Clinically Important Differences in the Edmonton Symptom Assessment Scale in Cancer Patients: A Prospective, Multicenter Study. Cancer. 2015;121 (17):3027-35.

15. Yennurajalingam $S$, Urbauer DL, Casper KLB, et al. Impact of Palliative Care Consultation team on Cancer-Related Symptoms in Advanced Cancer Patients 
Referred to an Outpatient Supportive Care Clinic. Journal of Pain and Symptom Management. 2011;41: 49-56.

16. Hui D, Shamieh O, Paiva CE, et al. Minimally Clinically Important Difference in Physical, Emotional, and Total Symptom Distress Scores of the Edmonton Symptom Assessment System. Journal of Pain and Symptom Management. 2016;1: 262-269.

17. Kwon JH, Hui D, Chisholm G, et al. Clinical Characteristics of Cancer Patients Referred Early to Supportive and Palliative Care. Journal of Palliative Medicine. 2013, 16(2): 148-155.

18. Rhondali W, Yennurajalingam S, Chisholm G, et al. Predictors of response to palliative care intervention for chronic nausea in advanced cancer patients. Support Care Cancer. 2013 Sep; 21(9): 2427-2435 